\title{
Limited B Cell Repertoire in Severe Combined Immunodeficient Mice Engrafted with Peripheral Blood Mononuclear Cells Derived from Immunodeficient or Normal Humans
}

\author{
Andrew Saxon, ${ }^{*}$ Eric Macy, ${ }^{*}$ Kathleen Denis, ${ }^{*}$ Magdelena Tary-Lehmann, ${ }^{*}$ Owen Witte, Jonathan Braun* \\ *Division of Clinical Immunology and Allergy, Hart and Louise Lyon Immunology Laboratory, Department of Medicine and \\ ${ }^{\ddagger}$ Department of Pathology, UCLA Medical School, Los Angeles, California 90024-1680; and ${ }^{\S}$ Howard Hughes Medical Institute \\ and the Department of Microbiology, UCLA, Los Angeles, California 90024-1489
}

\begin{abstract}
The ability to engraft human PBMC or fetal tissue immune cells in the severe combined immunodeficient (SCID) mouse has created a need for characterization of these systems and their application to disease models. We demonstrate that SCID mice reconstituted with PBMC support the growth and differentiation of a restricted set of $B$ cells. Human IgG levels of 1-2 $\mathrm{mg} / \mathrm{ml} \mathrm{(10-20 \%} \mathrm{of} \mathrm{normal} \mathrm{human} \mathrm{serum} \mathrm{levels)} \mathrm{were} \mathrm{routinely}$ achieved in spite of a serum half life of only $12 \mathrm{~d}$. Ig levels peaked around $50 \mathrm{~d}$ and $\mathrm{Ig}$ production was maintained for $>100$ d. The Ig was $>85 \%$ IgG though some IgM, IgA, IgD, and even IgE could be detected. However, the human IgG produced in hu-PBL-SCID mice was pauci-clonal when analyzed by isoelectric focusing and by kappa/lambda light chain usage. Using a new polymerase chain reaction based analysis capable of monitoring individual VH family utilization, we found that the engrafted B cells showed skewed and restricted human VH subfamily utilization. These parameters were markedly variable among hu-PBL-SCID mice reconstituted from the same donor cell population at both early (21-50 d) and late stages (> 100 d). Hu-PBL/CVI-SCID mice constructed with cells from patients with common variable immunodeficiency with an in vitro block in terminal $B$ cell differentiation produced human Ig responses that were quantitatively the same as those produced by hu-PBL-SCID mice from normal donors. The huPBL-SCID system using PBMC appears to lead to growth and Ig production by a small number of $B$ cells and results in a restricted B cell repertoire. (J. Clin. Invest. 1991. 87:658665.) Key words: human B cells • human humoral immunity • humoral immunodeficiency • human SCID mouse chimeras
\end{abstract}

\section{Introduction}

Recently, CB.17 severe combined immunodeficient (homozygous scid/scid, SCID) ${ }^{1}$ mice have been shown to accept xenogeneic transplants of human lymphoid tissue, specifically pe-

Address correspondence and reprint requests to Dr. Andrew Saxon, Division of Clinical Immunology/Allergy, 10833 Le Conte Avenue, 52-175 CHS, Los Angeles, CA, 90024-1680. Dr. Denis' current address is Specialty Laboratory, 2211 Michigan Avenue, Santa Monica, CA 90402.

Received for publication 24 July 1990 and in revised form 10 September 1990.

1. Abbreviations used in this paper: $\mathrm{CVI}$, common variable immunodeficiency; IEF, isoelectric focusing; PCR, polymerase chain reaction; SCID, severe combined immunodeficient; XLA, X-linked agammaglobulinemia.

\section{J. Clin. Invest.}

(c) The American Society for Clinical Investigation, Inc. 0021-9738/91/02/0658/08 $\$ 2.00$

Volume 87, February 1991, 658-665 ripheral blood mononuclear cells when administered intraperitoneally as well as whole tissue implants of human fetal liver, thymus, and lymph node (1-3). SCID mice have also been shown to be permissive to the growth of human hybridoma and whole tissue implants of human lung cancers $(4,5)$. When $10-$ $50 \times 10^{6}$ mature normal human PBMCs are injected intraperitoneally, total human Ig levels of $>1 \mathrm{mg} / \mathrm{ml}$ (an order of magnitude below normal human serum concentrations) have been demonstrated. Use of PBMC reconstitution of mice is simple to undertake and could be used to explore many basic and clinical questions relating to immune function. However, the diversity of this response in terms of B cell repertoire and Ig diversity needed to be characterized in order to ascertain the suitability of hu-PBL-SCID mice for modeling of human immune function.

We present evidence on the nature of the $B$ cell engraftment and expression of humoral immunity in hu-PBL-SCID mice constructed with cells from normal subjects and from patients with humoral immune defects that have been characterized both in vivo and in vitro. By determining the nature and kinetics of human Ig production in these animals and extending this model human humoral immunodeficiencies, we have been able to delineate the nature of the humoral reconstitution in the hu-PBL-SCID mouse system.

\section{Methods}

Subjects. Informed consent was obtained from normal control, common variable immunodeficiency (CVI), and X-linked agammaglobulinemia (XLA) individuals who served as blood donors for SCID mice reconstitutions. These studies were approved by both the UCLA Human Institutional Review Board and the UCLA Animal Welfare Committee. The normal donors were screened for EBV and HIV status, all were negative for HIV antibodies. Two of the normals shown in Fig. 1 were negative for antibodies to EBV nuclear and viral capid antigens. Individuals with CVI or XLA were not screened for EBV status but were evaluated for HIV status using the HIV specific polymerase chain reaction (PCR) method; all were nonreactive. None of the patients had active autoantibody mediated disease, hepatitis, gastrointestinal disease, or lymphoproliferative malignancy. These individuals with CVI or XLA fail to produce spontaneous high rate Ig-secreting lymphoblastoid B cells or measurable serum antibody responses to booster immunization (6) and produce very low levels of Ig in PWM-stimulated cultures. The immunodeficient patients CVI 1-4 and XLA 1-2 are UCLA CIA patients No. 111, 118, 109, 108, 140, and 141, respectively. All of the individuals with CVI or XLA are maintained on intravenous immunoglobulin but had pretreatment IgG levels of $<2 \mathrm{mg} / \mathrm{ml}$ compared with normal adult human levels $6-15 \mathrm{mg} / \mathrm{ml}$. CVI is a heterogeneous group of diseases with a spectrum of intrinsic B cell maturational defects and non-B cell regulatory abnormalities (7-9). Individuals with CVI have measurable serum IgG (10-20\% of normal levels) but lack high affinity antibodies (10). The CVI patients' cells studied have been previously shown to an in vitro intrinsic $B$ cell maturational defect in terminal differentiation in that, when stimulated, they would undergo RNA and DNA synthesis but did not transit to high rate Ig production 
except for CVI No. 4 whose circulating B cells fail to undergo activation measured by Ig RNA synthesis and size change (11). Circulating B cell were present in all the CVI subjects studied. The two patients (brothers) with XLA have no circulating B cells detectable as defined by membrane Ig, but one maintains IgA levels in vivo at $10 \%$ of normal.

SCID mice and cell transfer. CB.17 scid/scid (SCID) immunodeficient mice were bred at UCLA under sterile conditions and maintained in a pathogen free environment without antibiotics in the water. Mice were between 6 and 12 wk old at the time of cell transfer. PBMC were separated from human donors by Sepracel flotation. 10-20 $\times 10^{6}$ cells were injected intraperitoneally via a No. 25 needle into SCID mice in 0.5-1 ml of RPMI 1640 medium containing $10 \%$ fetal calf serum, $1 \%$ glutamine, and $0.1 \%$ gentamicin. No mice observed up to $170 \mathrm{~d}$ developed frank human lymphoid tumors under these conditions.

Serum half-life of human Ig in mice. Human gammaglobulin for intravenous administration (a gift from Alpha Therapeutics, Los Angeles, CA) was administered intraperitoneally at a dose of $50 \mathrm{mg}$ of human IgG in $1 \mathrm{ml}$ to four unreconstituted SCID mice (mean weight $\pm S D: 23.8 \pm 0.3 \mathrm{~g}$ ) that were then serially bled over a $28-\mathrm{d}$ period. Serum samples were frozen once, thawed simultaneously, and assayed for total IgG and tetanus toxoid-specific IgG levels. Half-life was determined by plotting the slope $(M)$ of the log of the serum concentrations versus time using the equation $t_{1 / 2}=-(\log 2) / M$.

Isoelectric focusing (IEF). Agarose-amphylyte IEF was done using the Food, Materials and Clothing Corp. (Marine Colloids Division, Rockland, ME) Isogel System with modifications as noted. Hu-PBLSCID mice sera were used undiluted and were derived from mice between 50-80 d after injection with human cells. Control sera from normal donors $\mathrm{N} 1$ and $\mathrm{N} 2$ was diluted 1:5 while intravenous gammaglobulin was diluted 1:10. Samples ( $7 \mu$ l except for CVI-4 and X-linked agammaglobulinemia where $14 \mu$ l were used) were spotted on the IEF gel to approximate equivalent amounts of IgG in each lane. The gel was made to focus proteins over a pH 3-10 range. The focused gel was blotted onto nitrocellulose paper and the paper was blocked with a $1 \%$ albumin solution. The banding pattern of $\operatorname{IgG}$ was revealed by localization with alkaline phosphatase-coupled anti-IgG identical to that used in the IgG ELISA. The color development was with p-nitrophenyl phosphate.

Human Ig measurements. Serum and cell culture Ig levels were measured in very sensitive ELISAs described in detail elsewhere (12). The specific assays used included total IgG, all IgG subclasses, IgM, IgA, IgD, IgE, kappa or lambda chain-specific isotype, and antigen-specific IgG for tetanus toxoid. The assays have very low backgrounds and are able to readily detect $<10 \mathrm{pg}$ of total isotype specific or antigen specific Ig.

Mouse blood for serum human Ig determinations was obtained by tail vein or retro-orbital collection. The mouse sera was diluted a minimum of 1:250, thus the minimum amount of human Ig detectable was $2.5 \mathrm{ng} / \mathrm{ml}$ for total isotype. Hu-PBL-SCID or hu-PBL/CVI-SCID sera was usually diluted between 1:25,000 and 1:250,000. Importantly, sera from either native SCID mice, SCID mice reconstituted with Balb/c spleen cells and having normal murine serum IgG levels, or normal $\mathrm{Balb} / \mathrm{c}$ mice produced no measurable human Ig signal even at a dilution of 1:20 in the ELISAs specific for human Ig employed.

Kappa/lambda ratios were determined by coating ELISA plates overnight with purified monoclonal heavy chain-specific anti-Ig of the appropriate isotype. The amount of human Ig bound was then identified with light chain-specific second antibodies, goat (Fab') 2 from Tago Immunochemicals (Burlingame, $\mathrm{CA}$ ). Control human AB serum routinely gave a kappa/lambda ratio $\sim 0.5,0.34 \mathrm{U}$ of kappa/0.66 $\mathrm{U}$ of lambda. No anti-double-stranded DNA antibodies were detected in any of the six Hu-PBL-SCID mouse sera analyzed.

Recovery of human cells from hu-PBL-SCID mice. Splenic, lung, and liver mononuclear cells were recovered by pulling apart the tissue in sterile medium and passing the fragments through sterile wire mesh. Bone marrow cells were flushed out of the femoral cavity. Peritoneal cells were obtained by lavage. Aliquots of cells were cultured at $100,000-1,000,000$ cells/ml for $14 \mathrm{~d}$ in RPMI 1640 containing $10 \%$ fetal calf serum, $1 \%$ glutamine, and $0.1 \%$ gentamicin.
Flow cytometric analysis. The method used to study lymphocytes by dual color flow cytometric analysis has been described previously (11). In these experiments, an FITC-conjugated pan human cell reagent directed against CD45 (Becton Dickinson Immunocytometry Systems, Mountainview, CA) was used to identify all human cells. Subsets of human cells were identified with several phycoerythrin-labeled reagents: $T$ cells with anti-CD3, CD4, and $C D 8$, while $B$ cells were assessed with anti CD19 and CD20 (all from Becton Dickinson Immunocytometry Systems), and anti-CD21 (B2; Coulter Immunology, Hialeah, FL). Mouse cells were distinguished by the presence of $\mathrm{H} 2-\mathrm{K}^{\mathbf{d}}$ (Pharmingen, San Diego, CA), which was biotinylated and detected with phycoerythrin conjugated streptavidin (Caltag Laboratories, San Francisco, CA). These antibodies demonstrated species specificity. In control experiments mixing various proportions of mouse spleen and human blood cells, we were able to detect human cells when they comprised $1 \%$ of the mixtures.

Analysis of human engraftment and $B$ cell $V H$ gene utilization by $P C R$. DNA was prepared from fresh human or hu-PBL-SCID tissues as previously described (13). Enzymatic amplifications were generally performed as described by Saiki et al. (14) using $100 \mathrm{ng}$ of predenatured ( $94^{\circ} \mathrm{C}, 2 \mathrm{~min}$ ) genomic DNA prepared from tissue samples, 1-2 U of thermus aquaticus DNA polymerase (Perkin-Elmer/Cetus Corp, Norwalk, CT), and 100 pmol of each primer per assay (100 $\mu$ l, total final volume). Radioactive amplification products were prepared by supplementing individual PCR assay mixture dNTP pools with $0.1 \mu \mathrm{Ci}$ of 32P-[a]dCTP (Amersham Corp., Arlington Heights, IL) (15). All PCR assay mixtures were subjected to 40 successive cycles consisting of heat denaturation $\left(94^{\circ} \mathrm{C}, 2 \mathrm{~min}\right)$, oligonucleotide annealing, and primer extension $\left(72^{\circ} \mathrm{C}, 1.5 \mathrm{~min}\right)$ using a programmable cyclic reactor (Ericomp, San Diego, CA). Oligonucleotide primer pairs were used at the following annealing temperatures: $\mathrm{VH}+\mathrm{JHKOR}, 51^{\circ} \mathrm{C} ; \mathrm{LDLr}, 49^{\circ} \mathrm{C}$; $N$-ras, $49^{\circ} \mathrm{C}$.

Amplified products were fractionated by agarose electrophoresis using either $2.5 \% / 1.5 \%$ Nu-sieve/SeaKem (Food, Materials and Clothing Corp.) in $1 \times$ TPE buffer (0.08 M Tris-phosphate, $0.008 \mathrm{M}$ EDTA) for $N$-ras or $1 \% / 1 \%$ Nu-sieve/LE agarose gels in $1 \times$ TAE (0.04 M Trisacetate, 0.002 M EDTA) buffer for LDLr and Ig VDJ. For quantitation, the agarose gels were dried, and autoradiographs were prepared as templates to excise gel slices bearing amplified bands; Cerenkov counts were measured using a scintillation counter (Beckman Instruments, Inc., Palo Alto, CA). Duplicate samples were typically processed, and reported as arithmetic means; the standard errors were $<10 \%$.

\section{Oligonucleotide primers used.}

Human Ig VDJ segments, 5'-dAGTGCCAACCGCCTCACAGG (JHKOR, noncoding strand of consensus JH segment) (16); 5'-dTCTGGGGCTGAGGTGAAGAAG (VH1N), 5'-CTGGTGGAGTCTGGGGGAGGC (VH3L), 5'-dCCAGGACTGGTGAAGCC (VH46N), 5'-dGTGCAGCTGGTGCAGTCT (VH30), and 5'dGTCCAGTGTGAAGTGCAG (VHCP4), representing various subfamily-specific coding-strand FRI segments $(17,18) ; 5^{\prime}$-dCCTCCACAGTGAGTCTCC (VH5N, coding strand of a VH5 5'-flanking sequence) (17). Human LDL receptor (exons 14 and 15) (19), 5'-dAGTGCCAACCGCCTCACAGG and 5'-dCCTCTCACACCAGTTCACTC. Common Murine/Human $N$-ras exon II segment ( $N$-ras) (20), 5'-dGAGACATGTCTACTGGACATCTT and 5'-dTACGCCCTGGTCATGTACGCGTG.

Statistical analysis. Immunoglobulin measurement data were analyzed using the ELISA Master program for MacIntosh written by Richard Deem, UCLA Department of Medicine. Statistical tests as noted were performed using the Statworks software package for the MacIntosh personal computer.

\section{Results}

Hu-PBL-SCID mice from normals and CVI patients produce high levels of IgG that rapidly turns over. The kinetics and isotype distribution of Ig production by hu-PBL-SCID mice constructed with cells from normals or subjects with humoral 
immunodeficiency was studied. Mice were reconstituted with $20 \times 10^{6}$ PBMC from normal individuals, four individuals with CVI and two brothers with XLA. IgG, IgM, and IgA could be identified in at least one serum sample of all SCID mice reconstituted with PBMC from both normals and all individuals with CVI. All four IgG subclasses were identifiable in huPBL-SCID mice from the four CVI subjects and from the normal donor hu-PBL-SCID mice tested (data not shown). A few normal reconstituted mice additionally produced measurable IgD and/or IgE. No hu-PBL/CVI-SCID mice produced measurable IgE, but a few produced IgD. Fig. 1 shows the mean quantity of human IgG in reconstituted animals over time. Each plotted point represents the mean serum $\operatorname{IgG}(\mu \mathrm{g} / \mathrm{ml})$ of at least six samples (two to four mice/subject and two to three samples/ mouse). IgG levels of $1-3 \mathrm{mg} / \mathrm{ml}$ were achieved in all the normal hu-PBL-SCID mice and in hu-PBL/CVI-SCID mice (total of 10 ) constructed from three CVI subjects that were previously shown to have an in vitro defect in terminal IgG production (CVI 1-3). While overall IgG levels fell after day 50, IgG production continued out past day 100 as evidenced by individual mice showing little or no decline in serum IgG level between days 50 and 120 . Levels $>1 \mathrm{mg} / \mathrm{ml}$ were the norm at day 100 . The kinetics of IgM and IgA production were essentially equivalent to that of $\mathrm{IgG}$, both being readily detectable by day 15 and rising to near maximal levels by $50 \mathrm{~d}$. Maximum serum concentrations of human IgM and IgA were $10-40 \mu \mathrm{g} / \mathrm{ml}$. Mice producing higher levels of IgG tended toward higher IgM and IgA production.

To verify that active human Ig secreting cells were still present in long term hu-PBL-SCID mice, which maintained high levels of human serum Ig, hu-PBL-SCID mouse spleen, and bone marrow cells were cultured for human Ig production Table I. shows that human Ig was spontaneously produced in cultures from both spleen and bone marrow cells. Both IgG and IgM was identified in all cultures in which in vitro Ig production occurred even though the level of human B cells was $<1 \%$. Peritoneal lavage cells at these late time points failed to make any human IgG or IgM.

Hu-PBL/CVI-SCID mice constructed with cells from CVI No. 4 showed an early and rapid decrease in Ig production after day 21 although there was persistance of $\operatorname{IgG}$ out to $100 \mathrm{~d}$ at levels 10 -fold lower than in the normal or other CVI reconstituted mice. Hu-PBL/XLA-SCID mice produced with PBMC from two brothers who lacked measurable circulating $B$ cells also showed abnormal reconstitution (Fig. 1). Sera from huPBL/XLA-SCID mice from one subject contained very low levels of Ig at day $15(10 \mu \mathrm{g} / \mathrm{ml} \mathrm{IgG,} 0.5 \mu \mathrm{g} / \mathrm{ml} \mathrm{IgM,} \mathrm{and} 5 \mu \mathrm{g} / \mathrm{ml}$ $\operatorname{IgA})$ and then decreased below the limit of detection $(<2.5$ $\mathrm{ng} / \mathrm{ml}$ ) by day 30 through day 100 . This XLA patient's serum contained small amounts of $\mathrm{IgA}(0.10 \mathrm{mg} / \mathrm{ml})$. The phenomena of IgA production in XLA has been previously reported in a patient who had detectable IgA in his serum yet failed to produce IgA specific plaques to Cowan I, anti-mu, PWM, or T cell replacing factors (21). Sera from hu-PBL/XLA-SCID mice constructed with cell from the other XLA brother failed to show any human Ig.

The half-life of human IgG in SCID mice was determined. Serum IgG half-life is inversely related to concentration and mice have a far more rapid turnover of IgG than humans (22). Unreconstituted SCID mice were injected intraperitoneally

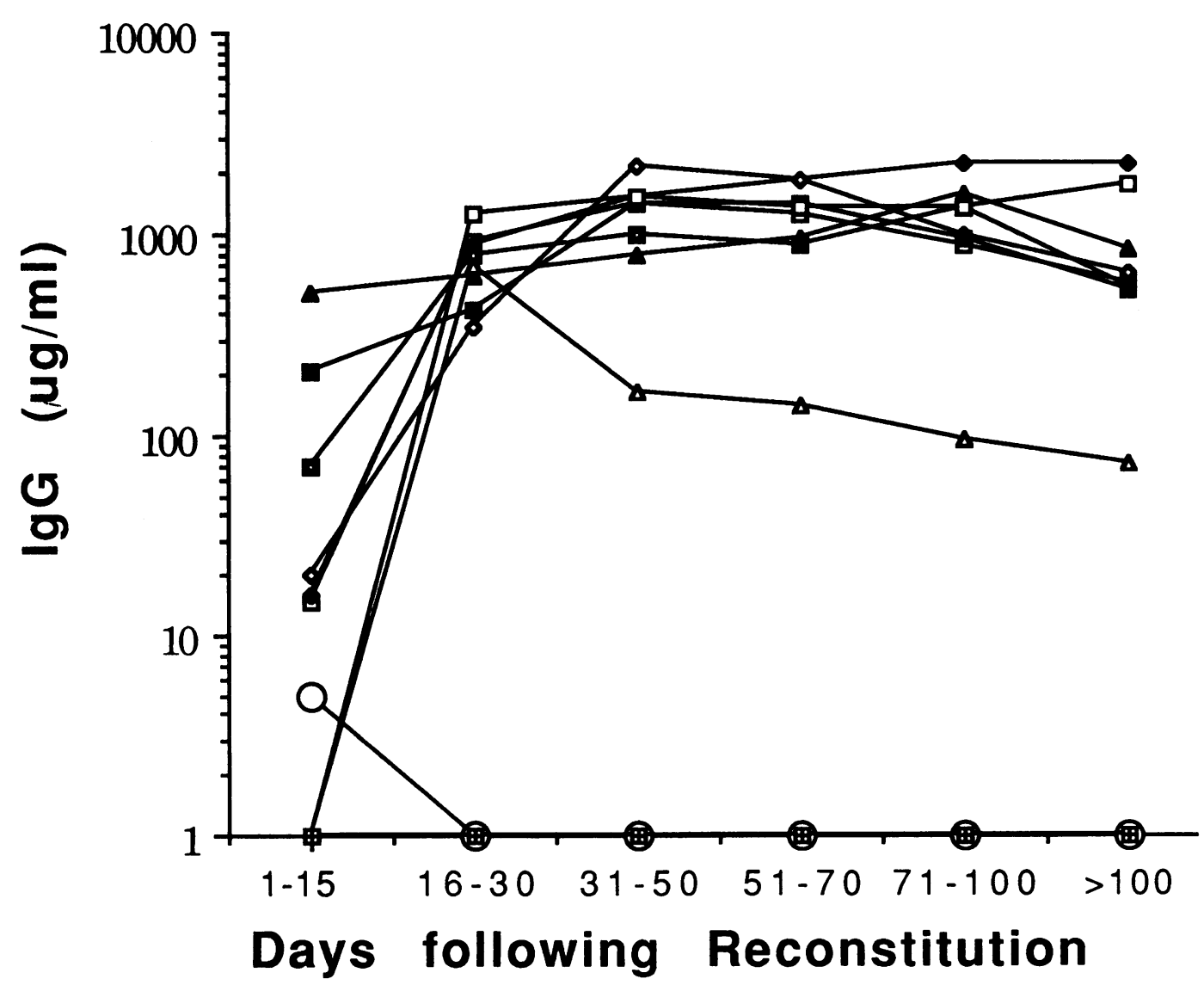

Figure 1. IgG levels in hu-PBL-SCID, hu-PBL/ CVI-SCID, and hu-PBL/ XLA-SCID mice. The mean quantity of circulating IgG versus time after reconstitution of the SCID mice is shown. The mean levels of IgG in 12 hu-PBLSCID mice from 4 normals are shown. Similar data for 19 SCID mice reconstituted with cells from 3 patients with CVI or 2 with XLA are shown. Each plotted point represents the mean serum IgG $(\mu \mathrm{g} / \mathrm{ml})$ of at least 6 samples $(2-4$ mice/subject and 2-3 samples/mouse). The number of animals represented for patients CVI-1, CVI-2, CVI-3, CVI-4, XLA-1, and XLA-2 are 3, 3, 4, 2, 4, and 3 , respectively. N1 $(-\bullet-)$; N2 (- -); N3 (-口); N4 (-৩-); CVI1 (-- - ) CVI2 (-口 -); CVI3 (-A-); CVI4 $(-\triangle-)$; XLA1 (-O-); and XLA2 (-⿴囗十). 
Table I. Human IgG and IgM Production In Vitro by Cells Derived from Long Term Reconstituted hu-PBL-SCID and hu-PBL/CVI-SCID Mice Compared with Serum IgG and IgM Levels in the Same Mice*

\begin{tabular}{|c|c|c|c|c|c|c|c|}
\hline \multirow[b]{3}{*}{ Mouse } & \multirow[b]{3}{*}{ Day } & \multicolumn{4}{|c|}{ In vitro production per $10^{5}$ cells cultured } & & \\
\hline & & \multicolumn{2}{|c|}{ Spleen } & \multicolumn{2}{|c|}{ Bone marrow } & \multicolumn{2}{|c|}{ Serum levels } \\
\hline & & IgM & IgG & IgM & IgG & IgM & IgG \\
\hline & & \multicolumn{4}{|c|}{$n g$} & \multicolumn{2}{|c|}{$\mu g / m l$} \\
\hline $\mathrm{N}-1 \mathrm{c}$ & 155 & 0.036 & 0.575 & 0.0 & 0.0 & 1.8 & 462 \\
\hline $\mathrm{N}-2 \mathrm{~b}$ & 91 & 10.6 & 106 & 9.6 & 166 & 133.6 & 651 \\
\hline CVI-1b & 112 & 0.16 & 28.6 & 0.08 & 0.215 & 1.7 & 543 \\
\hline CVI-2b & 112 & 0.66 & 2.5 & 0.08 & 0.185 & 30.3 & 1813 \\
\hline CVI-4a & 112 & 0.0 & 0.0 & 0.0 & 0.0 & $<0.01$ & 74 \\
\hline
\end{tabular}

* Simultaneous determination of spontaneous human Ig production over $14 \mathrm{~d}$ of culture by cells derived from spleen and bone marrow. The amounts shown are corrected to amount per $10^{5}$ cells. Concurrent serum IgG and IgM levels in the hu-PBL-SCID and hu-PBL/CVI-SCID mice is as shown. Peritoneal lavage cells failed in all cases to produce measurable Ig.

with $50 \mathrm{mg}$ of IgG of a well characterized human gammaglobulin preparation. This material has a half-life of $21 \mathrm{~d}$ in humans with normal IgG levels while the $t_{1 / 2}$ for IgG in humans with equivalent IgG levels to the hu-PBL-SCID mice would be over $60 \mathrm{~d}$. The $t_{1 / 2}$ of human IgG in SCID mice was $12 \mathrm{~d}$ for serum levels achieved in hu-PBL-SCID mice, while tetanus-specific IgG had a half-life of $5 \mathrm{~d}$ in the concentration given. Thus maintenance of the IgG levels observed in hu-PBL-SCID mice represents much more IgG synthesis than would be predicted from the $t_{1 / 2}$ in humans.

IgG produced in SCID-hu mice showed limited heterogeneity. The light chain representation in IgG and IgM produced by the hu-PBL-SCID and hu-PBL/CVI-SCID mice was used to assess the heterogeneity of Ig production (Table II). If the humoral reconstitution was truly polyclonal and reflected the normal in vivo situation in humans, the lambda/kappa ratio should be very near 0.5 (34\% lambda/66\% kappa) seen with normal human sera. In individual hu-PBL-SCID mice, was marked kappa or lambda predominance with ratios from 0.11 to 1.56 for IgG and from 0.03 to 5.06 for IgM, compared with the expected ratio of $\sim 0.5$ (34\% lambda/66\% kappa) (Table II). Skewing was present from the earliest (day 12) to latest (day 155) time of measurement. All but 4 of 36 samples (among 10 animals from 5 donors) fell outside a $0.4-0.6$ ratio range. The kappa/lambda ratio varied between mice reconstituted with the same normal donor cells in the same experiment.

To obtain an independent estimate of the heterogeneity of IgG present in the reconstituted SCID-hu mice, sera was isoelectrically focused over a 3-10 pH gradient. A pattern of up to 30 predominant bands was seen with hu-PBL-SCID and huPBL/CVI-SCID mice sera (Fig. 2) further suggesting restricted clonality of the IgG produced in these animals. In contrast, the normal human donors' sera or pooled human IgG are seen primarily as smears of IgG with rare bands being resolved on this background. The lack of IgG in hu-PBL/CVI-SCID No. 4 or hu-PBL/X-linked agammaglobulinemia/SCID mice sera was also confirmed.

The repertoire of engrafted $B$ cells in hu-PBL-SCID mice shows limited diversity. The degree of human B cell engraftment and clonal diversity was further characterized by quantitative PCR analysis of genomic DNA from SCID-hu mice (Fig. 3). Sense-strand primers were selected to detect subsets ("sub- families") of various VH gene families, and paired with an antisense-strand $\mathrm{JH}$ primer to specifically amplify only fully rearranged VDJ segments (23-26). Quantitative PCR reaction conditions were established for a range of 50-250 ng of tem-

Table II. Light Chain Usage in IgG and IgM from Sera of SCID Mice Reconstituted with Normal or CVI Subjects' Cells*

\begin{tabular}{lccc}
\hline $\begin{array}{c}\text { Mouse } \\
\text { No. }\end{array}$ & $\begin{array}{c}\text { Day serum } \\
\text { obtained }\end{array}$ & $\begin{array}{c}\text { IgG ratio } \\
\text { lambda/kappa }\end{array}$ & $\begin{array}{c}\text { IgM ratio } \\
\text { lambda/kappa }\end{array}$ \\
\hline N-1c & 43 & 0.72 & 0.74 \\
N-1c & 155 & 0.68 & 0.34 \\
N-1d & 33 & 0.90 & 0.43 \\
N-1d & 112 & 1.13 & 0.31 \\
N-2a & 51 & 0.83 & 4.16 \\
N-2b & 35 & 1.13 & 3.30 \\
N-2b & 76 & 1.56 & 5.06 \\
CVI-1a & 33 & 0.41 & 0.21 \\
CVI-1b & 33 & 0.64 & 0.07 \\
CVI-1b & 61 & 0.61 & 0.10 \\
CVI-1b & 112 & 0.48 & 0.07 \\
CVI-2a & 33 & 0.21 & 0.16 \\
CVI-2b & 33 & 0.25 & 0.51 \\
CVI-2b & 81 & 0.72 & 0.03 \\
CVI-2b & 112 & 0.89 & 0.12 \\
CVI-2c & 33 & 0.11 & 0.24 \\
CVI-3a & 12 & 0.39 & 0.36 \\
Normal human control serum 1 & 0.50 & 0.56 \\
Normal human control serum 2 & 0.53 & 0.46 \\
\hline
\end{tabular}

* The amount of isotype-specific kappa or lambda light chain associated immunoglobulin was determined by parallel ELISA assay developing one-half of each ELISA plate with specific anti-kappa second antibody and one-half with specific anti-lambda. Ratios were determined by coating ELISA plates overnight with purified monoclonal heavy chain specific anti-Ig of the appropriate isotype. The data shown represent optical densities which were on the linear portion of binding for the respective ELISA plate used. The normal control sera were obtained from the normal donors used to produce the normal hu-PBL-SCID mice used in this table. The expected ratio is $\sim 0.5$ as shown by the normal control sera. 

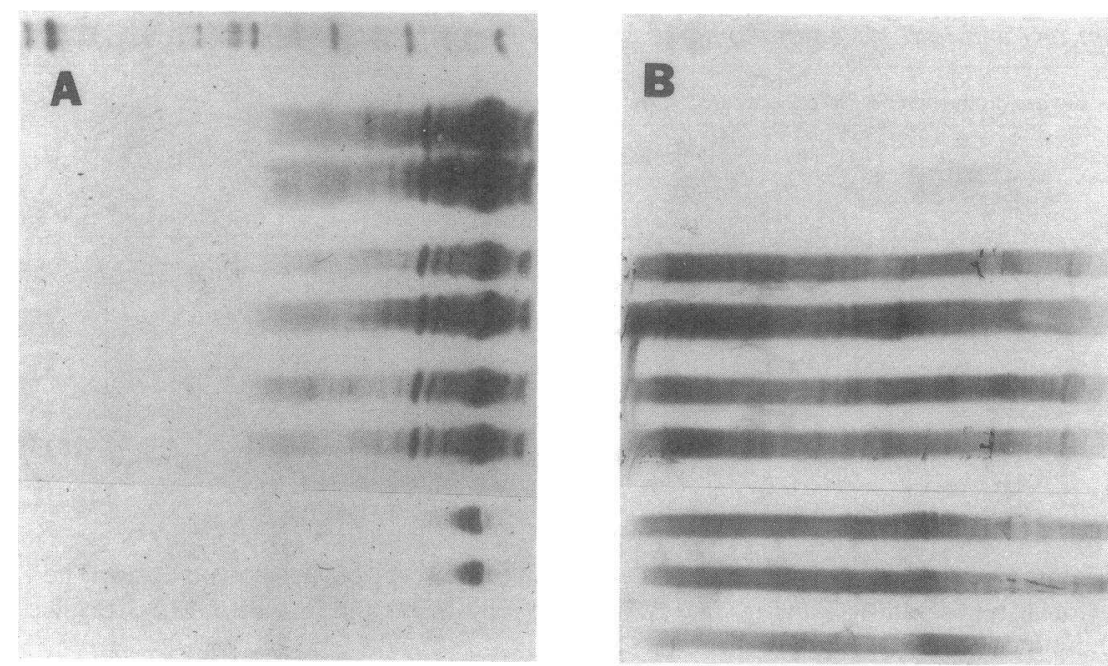

\section{Markers}

X-linked agammaglobulinemia

CVI-4

CVI-3

CVI-1

Normal-2

Normal-1

Normal serum \#2

Normal serum \#1

Intravenous Gammaglobulin

Figure 2. Isoelectric focusing of human IgG produced in reconstituted SCID mice. Hu-PBL-SCID mice sera, control sera from the normal human donors, and intravenous IgG were subjected to IEF over a pH range from 3 to 10. Equivalent amounts of IgG were run in all lanes except samples from CVI-4 and XLA to which maximum amounts of total protein were added. $(A)$ Commassie blue staining of proteins. The larger amounts of protein added for the hu-PBL-SCID mouse sera is apparent. $(B)$ IgG staining using a specific anti-human IgG antiserum. Multiple descrete bands can be seen in the samples from hu-PBL-SCID normal 1 and 2 as well as CVI 1 and 3. Little or no IgG is present in the samples from the hu-PBL/CVI-4-SCID or hu-PBL/XLA-SCID mice, respectively. The IEF markers range from 3.8 on the right to 10.2 on the left.

plate DNA (25-29). Because human B cells usually bear only one fully rearranged allele (30), the abundance of VDJ segments for a particular VH subfamily gene directly corresponds to the abundance of $\mathrm{B}$ cells in the population expressing that VH subfamily. In this case, an unbiased hu-PBL-SCID B cell engraftment would be expected to yield the same proportion of rearranged $\mathrm{VH}$ subfamilies as in the original human donor material used for engrafting.

At the early phase of reconstitution (days 21-50), human $(\mathrm{CD} 45+)$ cells were readily detectable in spleen, liver, and lung and present among bone marrow cells (see below). Nonetheless, the VDJ clonal pattern of engrafted B cells during this phase (day 35) was skewed and restricted compared with the donor cell population, and heterogeneous among different tissues (Fig. $3 a$ ). By day 164, the VH diversity of engrafted cells was further diminished with marked expansion of VH3L B cells (Fig. $3 b$ ).

Analysis of three replicate animals from an independent long term reconstitution (day 141) is shown in Fig. 3, $c-e$. The VDJ clonal pattern was divergent and restricted compared with the donor cell population (note the selective increase in VH46 $B$ cells), and also heterogeneous among the three animals (Fig. $3 c$ ). The abundance of human B cells in these animals was very low $(0.014-0.14 \%$, Fig. $3 d)$, despite persistently high levels of in vivo and in vitro production of human IgG (see above). The level of total human cell engraftment was of greater magnitude $(<0.5-4 \%$, Fig. $3 e)$, but not necessarily concordant from animal to animal with the level of B cell engraftment. Among several sets of long term ( $>120 \mathrm{~d}$ ) hu-PBL-SCID mice, a similar level of CD45 + human cells $(<1-3 \%)$ was detected in mononuclear preparations from spleen, lung, liver, and bone marrow.

The level of human cell reconstitution was confirmed in hu-PBL-SCID animals by flow cytometric analysis. CD45+ human cells were generally found in the hu-PBL-SCID mice spleen, liver, and lung (10-70\% range in 20 animals), being most readily detectable at days $35-50$ with less human cells being present in the bone marrow (1-5\%). There was gradual disappearance of these human cells over the ensuing months. Almost all of these human CD45 + cells were CD3 + T cells, the analysis of which will be reported elsewhere (Tary-Lehmann et al., in preparation). From a total of 20 animals, the maximum percentage of human cells that were B cells (CD19 and/or $\mathrm{CD} 20+$ ) was $10 \%$. In the majority of animals, $1 \%$ or less of the human cells were B cells at all time points (day 21 to $>100$ ). By day 120 , generally $<1 \%$ of the liver, lung, spleen, peritoneal lavage, and bone marrow cells recovered stained for CD3, CD4, CD19, CD20, Leu M3, or CD45. Rare human T cells ( $<1 \% \mathrm{CD} 3$ and either $\mathrm{CD} 4$ or $\mathrm{CD} 8$ positive) were observed on immunohistologic staining of hu-PBL-SCID splenic tissue. After $120 \mathrm{~d}$, no human $B$ cells were identifiable in either spleen or bone marrow sections despite high levels of in vivo human antibody production in these mice at the time cells were obtained for analysis (Table II). This data was corroborated by analysis of human repetitive Alu sequences in DNA extracted from the tissues of these same mice and by analysis of the LDL receptor/N-ras gene representation (Fig. 3 ).

\section{Discussion}

The ability to graft human immune tissues into CB.17 (SCID) mice holds great promise as a system for performing both long term culture and the in vivo manipulation of human cells (15 ). We explored the utility of the hu-PBL-SCID mouse model for the investigation of the normal human humoral immunity and for the deficiency state, CVI. Surprisingly, Ig production and diversification in SCID mice given PBMC from three CVI patients previously found incapable of making Ig in vitro (11) was indistinguishable from those given normal PBMC. $\mathrm{Hu}-$ PBL/CVI-SCID animals derived from an unusual CVI patient whose B cells failed to undergo activation in vitro only showed a brief human Ig production. 
(a) VDJ Clonal Pattern (Day 35)

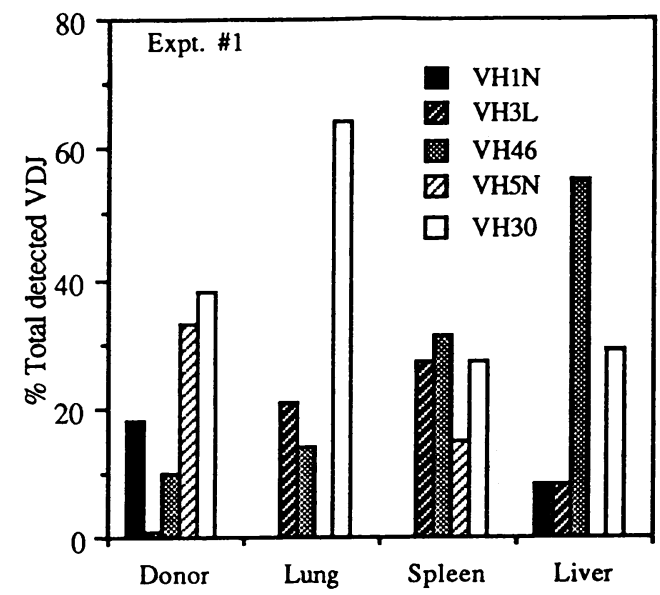

(b) VDJ Clonal Pattern (Day 164)

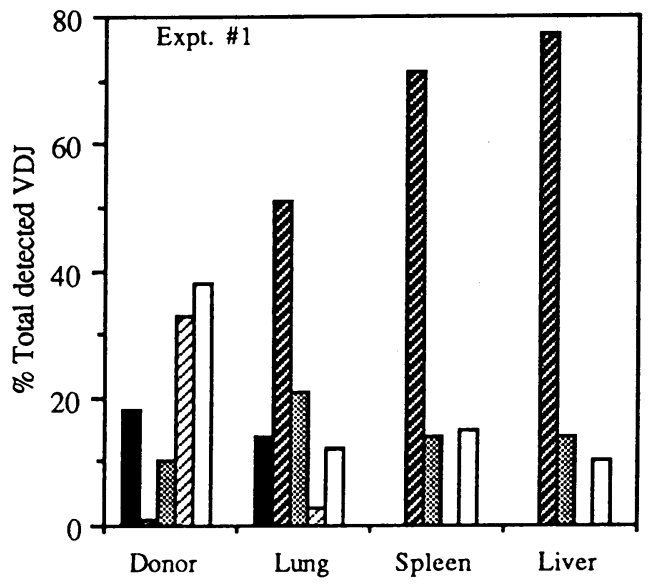

(c) VDJ Clonal Pattem (Day 141)
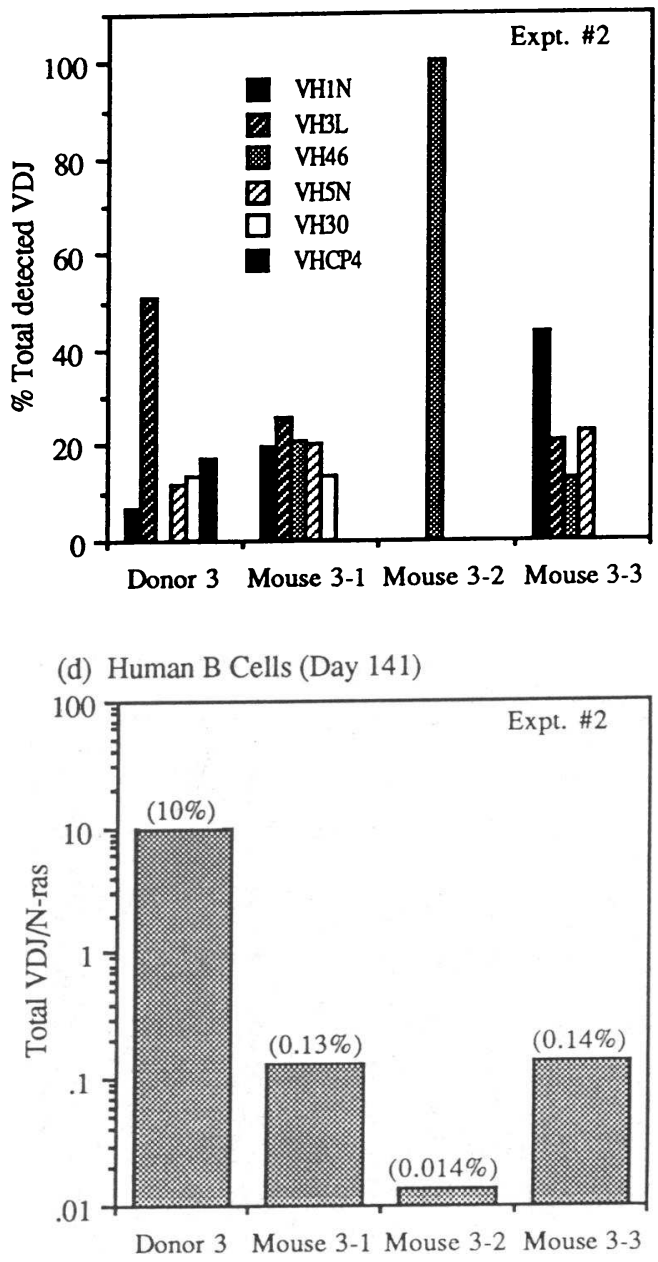

(e) Total Human Cells (Day 141)

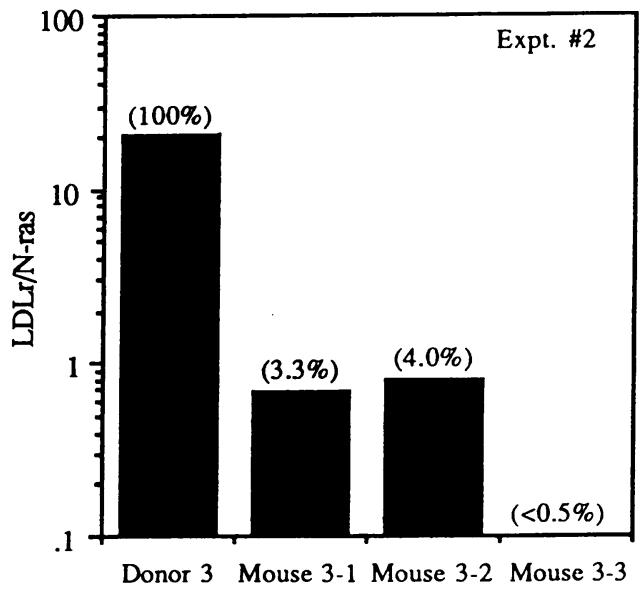

Figure 3. Genomic PCR analysis of human cell engraftment in hu-PBLSCID mice. $(a, b)$ VDJ clonal pattern in various tissues from hu-PBLSCID mice reconstituted with $10^{7} \mathrm{PMBC}$ for 35 and $164 \mathrm{~d}$ (Experiment 1). The relative abundance of rearranged VH subfamilies was determined for genomic DNA from the original donor cell population and from spleen, liver, and lung of hu-PBL-SCID mice. At day 35, CD45+ cells in mononuclear cells recovered from these organs was 32,17 , and $22 \%$, respectively; at day 164, CD45 + cells were 3,2 , and $28 \%$, respectively. $(c-e)$ Engraftment pattern for three hu-PBL-SCID mice (3-1, 3-2, and 3-3) constructed at the same time with $10^{7}$ cells of a normal donor cell population (same as $\mathrm{NI}$ in Fig. 1), and analyzed on day 141 (Experiment 2). (c) VDJ clonal pattern. (d) Human B cells (ratio of total detected VDJ to $N$-ras). Values in parentheses are the percentage of human $B$ cells calculated as (huPBL-SCID ratio)/(donor ratio) $\times 10 \%$, where $10 \%$ was the frequency of donor B cells determined by flow cytometry. (e) Total human cells (ratio of human LDLr to $N$-ras). Values in parentheses are the percentage of total human cells calculated as (hu-PBL-SCID ratio)/ (donor ratio) $\times 100 \%$. Oligonucleotide pairs consisting of a conserved human $\mathrm{JH}$ region and various VH subfamilyspecific primers were used at a limiting template concentration $(100 \mathrm{ng})$ to quantify in cpm the abundance of fully rearranged human VDJ genomic segments; values are expressed as a percentage of the total detected VDJ (summation of cpm from all VH subfamilies in each sample). Total detected VDJ was also used as a measure of total human B cells, and quantitative amplification of a human LDL receptor genomic segment (19) was used as a measure of total human cells. In the latter cases, values were normalized to total recovered genomic DNA using a shared murine/human region of the $N$-ras gene (20). Conditions for enzymatic amplifications and oligonucleotide primers have been previously described $(19,20,25)$. 
These results raised questions as to the nature of the B cell and supporting cells which provide for the Ig production observed in our hu-PBL-SCID animals. Our results indicate that the hu-PBL-SCID model as employed using PBMC predominantly selects for growth of small numbers of precursors of high rate Ig secreting cells. Mature B cells are required; SCID mice given PBMC from two patients with XLA who lack circulating $B$ cells failed to make human Ig, while hu-PBL/CVI-SCID mice constructed from three CVI subjects previously shown to have circulating $\mathrm{mIg}$ bearing $\mathrm{B}$ cells and an in vitro defect in terminal Ig production (CVI 1-3) had Ig levels indistinguishable from normals.

Thus the question arises as to whether the hu-PBL/CVISCID animals represent the induction of a "normal phenotype" in the SCID mouse environment or conversely, whether the normal hu-PBL-SCID mice are, in fact, manifesting a limited humoral immune phenotype/function which can be achieved by both normals and CVI subject cells. The limited and skewed repertoire of B cells, determined by rearranged VH gene families, strongly supports the latter concept as does the variation in VH family representation between animals from the same donor in the same experiment. It appears that a huPBL-SCID mouse represents the engraftment and expression of a small number of $B$ cell clones, clones that appear to be relatively randomly selected even within animals injected from a single donor at one point in time. The limited heterogeneity and light chain predominance of the Ig and the paucity of human B cells observed in the hu-PBL-SCID mice provides additional evidence to support this notion.

Our data suggest that the hu-PBL-SCID model, as presently employed using PBMC, has the limitation of selecting for the growth of a small number of potential precursors of high rate Ig secreting cells present in PBMC. Therefore such cells have only to be present in a limited number to achieve the present level of humoral reconstitution. While it would be theoretically possible to simply use smaller numbers of cells for reconstitution and thereby test the frequency of B cell precursors for engraftment, we and others (2) have noted that graft sizes below 5 $\times 10^{6}$ cells leads to overall failure to engraft human cells.

We have observed that it is possible to enhance specific IgG antibody produced in hu-PBL-SCID mice using donor immunization to induce spontaneous antibody (anti-tetanus toxoid) cells $(31)$ in the blood used for SCID mouse reconstitution. This would be equivalent to the use of blood from patients with primary biliary cirrhosis (32) or systemic lupus erythematosous (33), known to contain B cells spontaneously producing disease-related antibodies in vitro to engender such an antibody response in hu-PBL-SCID mice. We have characterized such spontaneous antibody producing cells in depth both in vitro and in vivo and have shown they represent a form of triggered memory cell that is capable of transferring both an adoptive antibody response and B cell memory in humans (34). Thus the present hu-PBL-SCID system using peripheral blood mononuclear cells has important quantitative as well as qualitative limitations that need to be overcome before normal immune function can be evaluated and used to investigate humoral immunodeficiency disorders that are less dramatic than X-linked agammaglobulinemia.

\section{Acknowledgments}

We thank our colleagues Dr. Dan Adelman for helping to provide CVI patient samples, Dr. Florence Hoffman for immunohistochemical stud- ies, Lily King for PCR measurements, Brigette Keld for isoelectric focusing and Dr. Bevra Hahn for performing the anti-DNA studies.

Dr. Macy was supported by a grant from the UC-AIDS Taskforce. Dr. Braun is a Scholar of the Leukemia Society of America, and recipient of an RJR-Nabisco Research Scholars Award. Dr. Witte is an Investigator of the Howard Hughes Medical Institute. Supported by the United States Public Health Service grants CA-12800, AI-15251, AI15332, CA-23175, and grants from the American Cancer Society (IM482) and the UCLA AIDS Clinical Research Center.

\section{References}

1. McCune, J. M., R. Namikawa, H. Kaneshima, L. D. Shultz, M. Lieberman, and I. L. Weissman. 1988. The Hu-PBL-SCID mouse: murine model for the analysis of human hematolymphoid differentiation and function. Science (Wash. DC). 241:1632-1639.

2. Mosier, D. E., R. J. Gulizia, S. M. Baird, and D. B. Wilson. 1988. Transfer of a functional human immune system to mice with severe combined immunodeficiency. Nature (Lond.). 335:256-259.

3. Mosier, D. E., R. J. Gulizia, S. M. Baird, and D. B. Wilson. 1989. On the SCIDs? Nature (Lond.). 338:211.

4. Ware, C. F., N. J. Donato, and K. Dorshkind. 1985. Human, rat or mouse hybridomas secrete high levels of monoclonal antibodies following transplantation into mice with severe combined immunodeficiency disease (SCID). J. Immunol. Methods. 85:353-361.

5. Reddy, S., D. Piccione, R. B. Bankert. 1987. Human lung tumor growth established in the lung and subcutaneous tissues of mice with severe combined immunodeficiency. Cancer Res. 47:2456-2460.

6. Stevens, R. H., M. Tamaroff, and A. Saxon. 1980. Inability of patients with common variable hypogammaglobulinemia to generate lymphoblastoid B cells following booster immunization. Clin. Immunol. Immunopathol. 16:336-348.

7. Saiki, O., P. Ralph, C. Cunningham-Rundles, and R. A. Good. 1982. Three distinct stages of B-cell defects in common varied immunodeficiency. Proc. Natl. Acad. Sci. USA. 79:6008-6012.

8. Mayer, L., S. M. Fu, C. Cunningham-Rundles, and H. G. Kunkel. 1984. Polyclonal immunoglobulin secretion in patients with common variable immunodeficiency using monoclonal B cell differentiation factors. J. Clin. Invest. 74:2115-2120.

9. Ashman, R. F., A. Saxon, and R. H. Stevens. 1980. Profile of multiple lymphocyte functional defects in acquired hypogammaglobulinemia, derived from in vitro recombination analysis. J. Allergy Clin. Immunol. 65:242-256.

10. Buckley, R. H. 1987. Immunodeficiency Diseases. JAMA (J. Am. Med. Assoc.). 258:2841-2850.

11. Saxon, A., J. V. Giorgi, E. H. Sherr, and J. M. Kagan. 1989. Failure of B-cells in common variable immunodeficiency to transit from proliferation to differentiation is associated with altered B-cell surface molecule display. J. Allergy Clin. Immunol. 84:44-55.

12. Macy, E., M. Kemeny, and A. Saxon. 1988. How to measure less than 10 picograms of a specific protein (immunoglobulin) in less than 8 hours. FASEB (Fed. Am. Soc. Exp. Biol.) J. 2:3003-3009.

13. Govan III, H. L., Y. Valles-Ayoub, and J. Braun. 1990. Fine mapping of DNA damage and repair in specific genomic segments. Nucleic Acids Res. 18:3823-3830.

14. Saiki, R. K., S. Scharf, F. Faloona, K. B. Mullis, G. T. Horn, H. A. Erlich, N. Arnheim. 1985. Enzymatic amplification of I-globin genomic sequences and restriction site analysis for diagnosis of sickle cell anemia. Science (Wash. DC). 230:1350-1354

15. Schowalter, D. B., and S. S. Sommer. 1989. The generation of radiolabeled DNA and RNA probes with polymerase chain reaction. Anal. Biochem. 177:9094.

16. Crescenzi, M., M. Seto, G. P. Herzig, P. D. Weiss, R. C. Griffith, and S. J. Korsmeyer. 1988. Thermostable DNA polymerase chain amplification of $t(14 ; 18)$ chromosome breakpoints and detection of minimal residual disease. Proc. Natl. Acad. Sci. USA. 85:4869-4873.

17. Berman, J. E., S. J. Mellis, R. Pollack, C. L. Smith, H. Suh, B. Heinke, C. Kowal, U. Surti, L. Chess, C. R. Cantor, and F. W. Alt. 1988. Content and organization of the human Ig VH locus: definition of three new VH families and linkage to the Ig CH locus. EMBO (Eur. Mol. Biol. Organ.) J. 7:727-738.

18. Braun, J., and L. King. 1989. Unique $V$ gene usage by B-Lyl cell lines, and a discordance between isotype switch commitment and variable region hypermutation. J. Mol. Cell. Immunol. 4:121-128.

19. Li, H., U. B. Gyllensten, X. Cui, R. K. Saiki, H. A. Erlich, and N. Arnheim. 1988. Amplification and analysis of DNA sequences in single human sperm and diploid cells. Nature (Lond.). 335:414-417.

20. Guerrero, I., A. Villasanto, V. Corces, and A. Pellicer. 1985. Loss of the normal N-ras allele in a mouse thymic lymphoma induced by a chemical carcinogen. Proc. Natl. Acad. Sci. USA. 82:7810-7814. 
21. Saiki, O., P. Ralph, C. Cunningham-Rundles, and R. A. Good. 1982. Three distinct stages of B-cell defects in common varied immunodeficiency. Proc. Natl. Acad. Sci. USA. 79:6008-6012.

22. Waldmann, T. A., and W. Strober. 1969. Metabolism of Immunoglobulins. Prog. Allergy. 13:1-110.

23. Sanz, I., P. Kelly, C. Williams, S. Scholl, P. Tucker, and J. D. Capra. 1989. The smaller human VH gene families display remarkably little polymorphism. EMBO (Eur. Mol. Biol. Organ.) J. 8:3741-3748.

24. Ward, E. S., D. Gussow, A. D. Griffiths, P. T. Jones, and G. Winter. 1989. Binding activities of a repertoire of single immunoglobulin variable domains secreted from Escherichia coli. Nature (Lond.). 341:544-546.

25. Valles-Ayoub, Y., H. L. Govan III, and J. Braun. 1990. Evolving abundance and clonal pattern of human germinal center $B$ cells during childhood. Blood. 76:17-24.

26. Schlissel, M. S., and D. Baltimore. 1989. Activation of immunoglobulin kappa gene rearrangement correlates with induction of germline kappa gene transcription. Cell. 58:1001-1007.

27. Becker-André, M., and K. Hahlbrock. 1989. Absolute mRNA quantification using the polymerase chain reaction (PCR). A novel approach by a PCR aided transcript titration assay (PATTY). Nucleic Acids Res. 17:9437-9446.
28. Frye, R. A., C. C. Benz, and E. Liu. 1989. Detection of amplified oncogenes by differential polymerase chain reaction. Oncogene. 4:1153-1157.

29. Syvanen, A. C., M. Bengtstrom, J. Tenhunen, and H. Soderlund. 1988. Quantification of polymerase chain reaction products by affinity-based hybrid collection. Nucleic Acids Res. 16:11327-11338.

30. Arnold, A., J. Cossman, A. Bakhshi, E. S. Jaffe, T. A. Waldmann, and S. J. Korsmeyer. 1983. Immunoglobulin gene rearrangements as unique clonal markers in human lymphoid neoplasms. N. Engl. J. Med. 309:1593-1597.

31. Stevens, R. H., E. Macy, C. Morrow, and A. Saxon. 1979. Characterization of a circulating subpopulation of spontaneous antitetanus toxoid antibody producing B cells following in vivo booster immunization. J. Immunol. 122:2498-2503.

32. Krams, S. M., K. Dorshkind, and E. M. Gershwin. 1989. Generation of biliary lesions after transfer of human lymphocytes into severe combined immunodeficient (SCID) mice. J. Exp. Med. 170:1919-1930.

33. Duchosal, M. A., P. J. McConahey, C. A. Robinson, and F. J. Dixon. 1990. Transfer of human systemic lupus erythematosus in SCID mice. J. Exp. Med. 172:985-988.

34. Saxon, A., R. Mitsuyasu, R. Stevens, R. E. Champlin, H. Kimata, and R. P. Gale. 1986. Designed transfer of specific immune responses with bone marrow transplantation. J. Clin. Invest. 78:959-968. 